\title{
ANALYZING INCONSISTENCY TOWARD ENHANCING INTEGRATION OF BIOLOGICAL MOLECULAR DATABASES*
}

\author{
YI-PING PHOEBE CHEN ${ }^{1,2}$ AND QINGFENG CHEN ${ }^{1}$ \\ ${ }^{1}$ Faculty of Science and Technology, Deakin University, Melbourne, VIC 3125, Australia, \\ ${ }^{2}$ Australia Research Council Centre in Bioinformatics, Australia
}

\begin{abstract}
The rapid growth of biological databases not only provides biologists with abundant data but also presents a big challenge in relation to the analysis of data. Many data analysis approaches such as data mining, information retrieval and machine learning have been used to extract frequent patterns from diverse biological databases. However, the discrepancies, due to the differences in the structure of databases and their terminologies, result in a significant lack of interoperability. Although ontology-based approaches have been used to integrate biological databases, the inconsistent analysis of biological databases has been greatly disregarded. This paper presents a method by which to measure the degree of inconsistency between biological databases. It not only presents a guideline for correct and efficient database integration, but also exposes high quality data for data mining and knowledge discovery.
\end{abstract}

\section{Introduction}

In recent years, advanced experiment methods have resulted in the rapid growth of life science databases. Many biological databases have been developed for different purposes, such as GenBank and NCBI [1-2]. The enormous data in databases are meaningful for the exploration of their life origin and evolution, and to predict the function and structure of life systems. They have been commonly usedby biologists during data analysis.

Due to the increasingly complex and specific nature of biological databases, a complicated biological question has to be answered by consulting multiple biological databases. However, the knowledge of life systems is too detailed and complex to be completely comprehended. Such complexity presents a big challenge to merge knowledge from diverse databases. The heterogeneity of databases blocks the accessibility to them [3-4]. In other words, the inconsistent structures and terminologies of biological databases result in a significant lack of interoperability. Thus, it creates a demand for data preprocessing.

As an important cleaning action, the integration of biological databases is significant when dealing with the heterogeneity of biological databases. However, the twisted and deformed biological data often demand additional knowledge so that the values held in databases can be specified and constrained. This causes considerable difficulties for data integration.

\footnotetext{
* This work is partially supported by a Discovery Grant DP0559251 from the Australian Research Council.
} 
Technical and semantic problems are two key issues which present themselves when integrating biological databases. The former can be solved because most current biological databases are implemented on relational database management systems (RDBMS) that provide standard interfaces like JDBC and ODBC for data and metadata exchange [5-6]. Nevertheless, the solution of semantic problems remains unsolved..

Modern bioinformatics demand knowledge extracted from databases for communication purposes. For example, a user's query of a protein kinase may refer to hundreds of databases. There are two options to integrate knowledge from databases: (1) standardising the nomenclature of diverse databases; and (2) creating bridges between databases even if they differ radically in structure and nomenclature. The former have encountered resistance from database maintainers and specialists who hesitate to change preferred terminology [4]. As a tradeoff scheme, the latter has been commonly applied in the integration phase of biological databases. Among them, ontology-based biological database integration is one of the representative methods designed to capture knowledge from databases.

There have been many attempts to develop standards that can be applied tobioinformatics ontologies and which subsequently exploit biological information. For example, EcoCyc ontology [7] covers E.coli gene, metabolism, regulation and signal transduction, and Gene ontology (GO) [8] describes drosophila, moused and yeast gene function, process and cellular location and structure. Recently, ontology-based semantic integration of biological databases was presented in [2, 9]. Philippi [6] proposed a method for the ontology-based semantic integration of life science databases using XML technology. To enhance semantic interoperability, there have been considerable efforts to solve nomenclature-mapping problems and standardise the naming of functional relations and processes and their arguments such as ontology-mapping in GO community [10]. It provides a comprehensive list of synonyms that can be used immediately to improve indexing and search over the literature. However, no effort has been made to analyse the inconsistency of biological databases which would effectively lead to the enhancement of database integration.

This paper presents a method by which to analyse inconsistencies between biological databases using ontology. The method is able to find out the databases that are inappropriate for integration or need to be further improved. This not only reduces the search space but also generates high quality data for accurate and efficient data mining and knowledge discovery. Algorithms and experiments are presented to further demonstrate our approaches.

The remainder of this paper is organised as follows. Section 2 presents basic concepts. The approaches by which to analyse the inconsistency between biological databases are presented in Section 3. In Section 4, experiments are presented. Section 5 concludes this paper. 


\section{Basic Concepts}

\subsection{Problem Description}

The increasing biological databases relating to genome sequences and protein structures and functions are challenging the traditional approaches for knowledge acquisition. To answer a complex biological question, hundreds of biological databases can be consulted. It is critical to guarantee accessibility to the databases. However, the discrepant structures and nomenclature of databases have an effect on their communication capabilities.

Although some biological data publishing and collection use HTML (Hypertext Markup Language) format, this method cannot describe complex structure documents. Besides, the varied organisation, storage and publication of biological data leads to different information types. For example, the representative database NCBI (National Center for Biotechnology Information) adopts mostly the binary ASN.1 [1], while flatfiles are used in GenBank [2]. The differences in the information types result in heterogeneities between biological databases and prevent us from obtaining high quality data for data analysis. Additionally, the information derived only from a single database does not enable us to obtain a comprehensive understanding, and the knowledge acquisition is inconvincible.

There have been some efforts to establish a link between disparate databases, such as data warehousing and database federation. Nevertheless, the increasing new data and databases have led efforts to reach a terminological impasse whereby databases have to agree on nomenclature and compatible formats before a link is able to be built. Nevertheless, database maintainers and specialists in certain research fields find it difficult to accept such a link. Ontology has been recently used to create bridges between biological databases. However, there are still some problems in the ontology-based integration of biological information. Problems include:

- Ontologies with independent terminologies and structures are often incompatible. This causes difficulties when acquiring knowledge from databases.

- Heterogeneities such as synonym result in a significant lack of interoperability among biological databases. This blocks the generation of high quality data.

- Semantic inconsistency has been widely ignored. The integration of biological databases with high discrepancies cannot guarantee efficient data mining.

Such inconsistencies surrounding biological databases and when the databases are appropriate for further processing, present major ambiguities. The analysis of the inconsistent nature of biological databases assists us in sorting out the appropriate databases from which high quality data can be derived. Hence, it is imperative to develop approaches by which to measure the inconsistency of biological databases and ensure reliable integration of biological databases.

\subsection{Symbols and Formal Semantics}

Suppose $A$ and $L$ denote atom symbols and proposition formulae respectively. In 
particular, $A$ can contain $\alpha$ and $\neg \alpha$ for some atoms $\alpha$. Let $\wedge$, $\neg$ and $\rightarrow$ be logical connectives. Let $C, c \in A$ be concepts such as Gene and Protein, $C V$ for control vocabulary, $r$ for relationship, and $\alpha, \beta$ and $\gamma$ for attributes in general. Let $\equiv$ be logical equivalence. A model of a formula $\phi$ is a possible set of atoms where $\phi$ is true in the usual sense.

Controlled vocabularies are a set of named concepts that may have an identifier. The concepts or their identifiers are often used as database entries. Its definition is as follows.

DEFINITION 2.1. Suppose $t$, def, id and sn present term, definition, identifier and synonyms respectively. Let $C$ be the set of concepts of databases. Hence, we have

$$
\text { Controlled Vocabulary } C V:=\{c \mid c=(t, \text { def, id, sn }) \in C\}
$$

An example of Gene Ontology (GO) is as follows. Each concept (biological process) has a term (recommended name), an identifier (id: GO: number), definition (explanation and references) and synonyms (other names). The definition of each biological process is provided by brief description and references to relevant literature or web links.

Ontology includes relationships as well as concepts. The relationships consist of 'isa' (Specification relationships) and 'part-of' (Partitive relationships), by which concepts can correlate with each other. Although 'part-of' relationship can be defined, only the transitive 'is- $a$ ' hierarchy is required for querying databases. For example, 'Enzyme is one kind of Protein', 'Protein is one kind of Macromolecule' and 'Membrane is part of Cells'. Therefore, ontology can be viewed as a tree, where the nodes and directed edges present concepts and relationships respectively.

DEFINITION 2.2. Let $O$ be ontology, and $r$ be relationships that link concepts. Ontology can be defined as a set of tuples.

$$
\text { Ontology } O:=\left\{\left(c_{1}, c_{2}, r\right) \mid c_{1}, c_{2} \in C V \text {, and } r: c_{1} \rightarrow c_{2}\right\}
$$

where $c_{1} \rightarrow c_{2}$ presents a relationship $r$ from $c_{1}$ to $c_{2}$, such as ' $c_{1}$ is- $a c_{2}$ '.

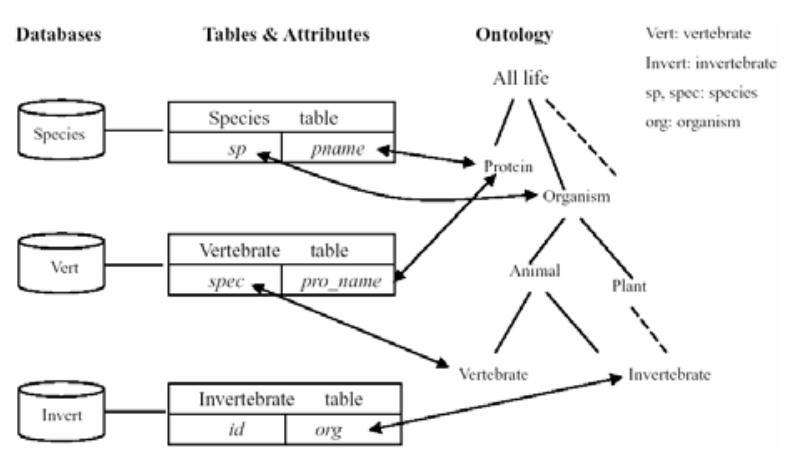

Figure 1. Biological database attributes are linked to ontology concepts. Attributes pname and pro_name from databases Species and Vert have different attribute names, but they are correlated by a common concept protein of the ontology.

EXAMPLE 2.1. In Figure 1, Vertebrate, Animal, Plant and Organism are connected 
by transitive 'is-a' relation. (Animal, Organism, Animal $\rightarrow$ Organism), (Plant, Organism, Plant $\rightarrow$ Organism), (Vertebrate, Animal, Vertebrate $\rightarrow$ Animal) and (Invertebrate, Animal, Invertebrate $\rightarrow$ Animal) represent tuples of ontology.

To analyse the inconsistency of biological databases, the above need to be defined semantically using ontology. One of the key processes is to link tables and attributes to a specified ontology. Subsequently, users can execute queries via hierarchies, such as 'is$a$ ', to derive information from databases. Four operators to describe the interactions among attributes, tables and ontology are given below. Let $A t t_{1} \in D B_{1}$ and $A t t_{2} \in D B_{2}$ be database attributes. Let $C V_{1}$ and $C V_{2}$ be controlled vocabularies.

(1) Mapping: Let Att $\in D B$ be database attributes. Let $O$ and $c$ be ontology and concepts respectively. 'maps $(O, A t t, c)$ ' states the attribute Att in $D B$ can be mapped to a corresponding concept $c$ via ontology $O$.

(2) Cross-reference: Let $C V$ be controlled vocabulary. 'cross-reference( $C V,\left(A t t_{1}, A t t_{2}\right)$,


reference of $C V$, they are semantically equivalent owing to $c$.

(3) Translation: 'translates $\left(\left(C V_{1}, C V_{2}\right),\left(A t t_{1}, A t t_{2}\right), c\right)$ ' states that database attribute $A t t_{1}$ and $A t t_{2}$ can be translated to a common concept $c$ using the controlled vocabularies $C V_{1}$ and $C V_{2}$. Thus, it is feasible to relate database entries that use different terms for the same thing, such as the English species name and Systematic species name in Figure 2.

(4) Taxonomy: Let $c_{i}$ and $c_{j}$ be concepts. ' $i s-a\left(c_{i}, c_{j}\right)$ ' states that $c_{i}$ is a sub-concept of $c_{j}$ and $c_{j}$ is a sup-concept of $c_{i}$. For simplicity, the operator ' $i s-a$ ' below implies both 'is- $a$ ' and 'part-of' relationships mentioned above. Actually, the 'is- $a$ ' relationship holds transitivity. Hence, we have

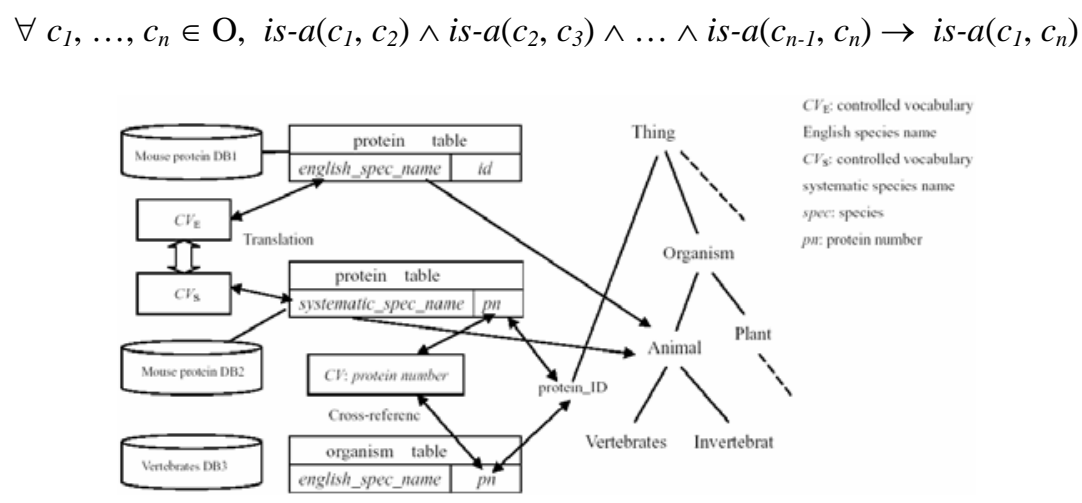

Figure 2. Translation by mapping synonymous concepts of controlled vocabularies is used to link databases with synonyms. Database attributes corresponding to the same concept and sharing the same controlled vocabulary can be viewed as cross-references of attributes.

The above axioms describe possible processes in response to a user's query on biological databases. Ontology plays a central role in mapping database attributes to common concepts or translating attributes between different controlled vocabularies, such as English controlled vocabulary and Systematic controlled vocabulary in Figure 2. 
Additionally, queries operator usually intends to search in attribute for specified terms as mentioned above. Hence, a user's query can be classified into two categories in terms of entries regarding attribute:

- if the queried attribute is found in databases, it will be mapped to a corresponding concept of ontology, and will enable other database attributes to be linked together;

- $\quad$ if no database attribute is defined as the queried attribute, a corresponding concept of ontology is selected. Its sub-concepts and super-concepts will be searched to find the attribute.

Although the latter is complex, it can eventually get back to the former pathway via ontology. In either case, the queries bring about a collection of results, which can be used to measure the inconsistency found in biological databases. Usually, users specify a term $T$ along with queries. $T$ is able to reduce the searched concepts that are irrelevant to the queries. Suppose Att is the queried attribute by users, and its mapping concept of ontology $O$ is $C$. Hence, we have

1. $\operatorname{sub}(C, T)=\{c \mid \forall c$, is- $a(c, C), c \sqsupseteq T\}$

2. $\sup (C, T)=\{c \mid \forall c$, is- $a(C, c), c \sqsupseteq T\}$

where $\sqsupseteq$ denotes a inclusion relationship in view of semantics.

EXAMPLE 2.2. Suppose a queried database attribute is Animal with a specified term parrot. Hence, in Figure 1, we have sub(Animal, parrot $)=\{$ Vertebrate $\}$, sup(Animal, parrot $)=\{$ Organism $\}$. Without the term parrot, sub(Animal, parrot $)=\{$ Vertebrate, Invertebrate $\}, \sup ($ Animal, parrot $)=\{$ Organism $\}$.

From the observation, the database attributes should be semantically defined as specific as possible, which can avoid searching unrelated databases.

DEFINITION 2.3. Let $A T T_{D B}=\left\{a_{1}, a_{2}, \ldots, a_{n}\right\}$ be a set of attributes of biological database $D B$. The set of attributes derived from reference database and compared databases are denoted by $A T T_{R}$ and $A T T_{C}$ respectively.

The reference database consists of multiple databases containing the queried attribute or the attribute that can be mapped to concepts of $\operatorname{sub}(C, T) \cup \sup (C, T)$. It is used to decide whether or not the attributes found in compared databases are consistent with the specified attribute. An example regarding $A T T_{R}$ and $A T T_{C}$ is given below.

EXAMPLE 2.3. In Figure 1, $A T T_{\text {Species }}=\{s p$, pname $\}, A T T_{\text {Vert }}=\left\{p r_{-}\right.$name, spec $\}$ and $A T T_{\text {Invert }}=\{i d$, org $\}$. If users query attribute pname, then $A T T_{R}=A T T_{\text {Species }}=\{s p$, pname $\}$ and $A T T_{C}=A T T_{\text {Vert }} \cup A T T_{\text {Invert }}=\{$ pro name, spec, id, org $\}$.

DEFINITION 2.4. Let $\vDash$ be a supporting relationship. For a set of database attributes $A T T_{D B}, A T T_{D B} \vDash$ is defined as follows.

(1) if the queried database attribute $\alpha$ is found in current databases, we have

- $\quad A T T_{R} \vDash \alpha$ iff $A T T_{R}$ contains $\alpha$

- $\quad A_{T} \vDash \neg \alpha$ iff $A T T_{c}$ contains $\beta$ that is a database attribute of compared databases, which has a common concept with $\alpha$.

(2) if the queried database attribute $\alpha$ is not found in databases but can be mapped to a concept $C$ in ontology, we have

- $\quad \operatorname{ATT}_{R} \vDash \alpha_{1}$ iff $\operatorname{ATT}_{R}$ contains $\alpha_{1}$ and $\operatorname{maps}\left(O, \alpha_{1}, c\right)$ 
- $\quad A T T_{C} \vDash \neg \alpha_{1}$ iff $A T T_{c}$ contains $\alpha_{2}$ that is the corresponding database attribute of $c$ in compared databases

Here $O$ and $c$ present the ontology and concepts in $\operatorname{sub}(C, T) \cup \sup (C, T)$ respectively. $\alpha_{1}$ denotes a mapping attribute in reference database from $c$.

EXAMPLE 2.4. Suppose 'pname : mouse' and 'animal : mouse' are two queries on Figure 1, in which pname and animal are queried attributes, and mouse is a term that locates databases. For the query 'pname : mouse', database Species can be viewed as the reference database. The term mouse reduces the search space to database Species and Vert. Hence, we have $A T T_{\text {Species }} \vDash$ pname and $A T T_{\text {Vert }} \vDash \neg$ pname. For the query 'animal : mouse', no database attribute is defined as animal. Nevertheless, the sub-concepts and super-concepts of animal in ontology can be mapped to this attribute. The search will be limited to Vertebrate and Species due to the term mouse that is mapped to attribute spec of Vert and sp of Species. Vert is selected as the reference database so $A T T_{\text {Vert }} \vDash$ spec. The attribute $s p$ of Species is viewed as a negative attribute of $s p e c$, namely $A T T_{\text {Species }} \vDash$ $\neg$ spec.

\section{Analyzing Inconsistency of Biological Databases}

\subsection{Models of Queried Biological Databases Attributes}

DEFINITION 3.1. Suppose ATT $\in \wp(L), X \in \wp(A)$. Let $A T T_{D B}$ be attributes derived from $D B \in\{R, C\}$. Let $X \vDash A T T$ denote that $X \vDash \alpha$ holds for every $\alpha$ in ATT.

$$
\text { model }(A T T)=\{X \in \wp(A) \mid X \vDash A T T\}
$$

where $A T T$ denotes a set of database attributes. The model of ATT actually presents a set of atoms that support ATT.

For measuring inconsistency, we use compatibility of biological databases. The consistentset of a model is the set of database attributes that have identical names with corresponding reference attributes. The conflictset of a model consists of (1) the set of database attributes that are semantically equivalent; and (2) the null attribute that presents no attribute is semantically equivalent to the reference attribute. Actually, some databases may not contain the queried attribute at all.

DEFINITION 3.2. Let $\delta$ be a selected reference attribute from reference database $R$. Let $Y \in \wp(A)$ be a model of $\delta$. The consistentset and conflictset are defined below.

- Consistentset $(\alpha)=\{\alpha \mid \alpha \in Y, \alpha \equiv \delta\}$

- $\operatorname{Conflictset}(\alpha)=\{\alpha \mid \alpha \in Y, \alpha \equiv \neg \delta$, or $\alpha \equiv$ null $\}$

Based on consistentset and conflictset from minimal models, a measurement can be used to compute the inconsistency of minimal models in respect to specified database attributes.

DEFINITION 3.3. The compatibility function from $A$ into $[0,1]$, is defined below when $\alpha$ is not empty, and Compatibility $(\varnothing)=0$.

$$
\operatorname{Compatibility}(\alpha)=\frac{\mid \text { Consistenset }(\alpha) \mid}{\mid \text { Consistentset }(\alpha)|+| \operatorname{Conflictset}(\alpha) \mid} \times 100 \%
$$


where $\mid$ Consistentset $(\alpha) \mid$ and $\mid$ Conflictset $(\alpha) \mid$ are the cardinality of Consistentset $(\alpha)$ and Conflictset $(\alpha)$ respectively. If Compatibility $(\alpha)=0$, then we can say that the model $Y$ has no opinion upon $\alpha$ and vice versa; if Compatibility $(\alpha)=1$, it indicates that there are no negative attributes $\neg \alpha$ in the model $Y$; if $0<\operatorname{Compatibility}(\alpha)<1$, it presents the model $Y$ as partially inconsistent/consistent with respect to $\alpha$.

The compatibility function quantifies the inconsistency of biological databases in relation to the queried database attributes. A queried attribute in biological databases is regarded as compatible or consistent in the case that the compatibility regarding this attribute is equal to, or greater than, the threshold minimal compatibility (mincomp) given by users or experts. Here, let mincomp $=0.5$. Then

- $\quad$ consistent if Compatibility $(\alpha) \geq \operatorname{mincomp}$

- $\quad$ inconsistent if Compatibility $(\alpha)<$ mincomp

Ideally, we would like to achieve Compatibility $(\alpha)=1$. Nevertheless, the inconsistency is the objective existence of discrepant terminologies and ontology used in diverse biological databases. If biological databases are found to be inconsistent, it is possible that they contain too many incompatible database attributes or most of current databases do not contain the queried database attribute. Therefore, the method presented in this paper is a prerequisite for the integration of biological databases.

\subsection{Experiments}

Table 1 represents the definition of attribute fields for five biological databases and refers to Gene ontology and NCBI databases. Among them, $D B_{2}$ and $D B_{5}$ are databases which regard Species, $D B_{1}$ and $D B_{4}$ are databases with respect to Vertebrate, and $D B_{3}$ is about Invertebrate. All database attributes under DNA sequence are linked to DNA sequence concept of the ontology. For the attributes under Organism, org is related to Vertebrate concept but spec is linked to Organism concept. The null value in this table means no such attribute is defined in corresponding biological databases, such as attributes of $D B_{1}$ under Description column. In particular, the attribute spec $_{s}$ of $D B_{5}$ is a systematic species name. A translation is, therefore, needed to search for this attribute.

Table 1. Attributes of biological databases.

\begin{tabular}{lcccccc}
\hline DB & Author & DNA Sequence & Description & Identifier & Organism & Enzyme \\
\hline $\mathrm{DB}_{1}$ & $a u$ & dns & null & id & org & null \\
$\mathrm{DB}_{2}$ & $a u t h o r$ & seq_dna & desc & id & spec & ename \\
$\mathrm{DB}_{3}$ & $a u$ & seq_dna & desc & mid & org & ename \\
$\mathrm{DB}_{4}$ & $a u$ & seq_dna & null & gid & spec & enzyme \\
$\mathrm{DB}_{5}$ & $a u$ & seq_dna & null & id & org & ec_nr \\
\hline
\end{tabular}

Measuring the inconsistency of biological databases mainly comprises of three steps: (1) input queried database attributes; (2) compute the compatibility of databases in relation to queried attributes; and (3) determine the consistency of databases. Two experiments are presented below. One is to query attribute 'enzyme : mouse' via crossreference, and the other is to query attribute 'animal : mouse' using translation.

In the former, $D B_{3}$ is ignored for it does not meet the constraint mouse. $D B_{5}$ is selected because the reference database for enzyme is found in $D B_{5}$, which is mapped to concept protein of the ontology in Figure 1. According to the ontology, the attributes 
under Enzyme of $D B_{2}, D B_{4}$ and $D B_{5}$ use different terminology to represent the same concepts. The common concept Enzyme can be used for cross-reference among them. According to Definition 3.2, we can obtain Consistentset (enzyme) $=\{$ enzyme $\}$ from $D B_{5}$, and Conflictset(enzyme $)=\{$ null, enmae, ename $\}$ from $D B_{1}, D B_{2}$ and $D B_{4}$. Both null and ename are regarded as $\neg$ enzyme when computing the compatibility of biological databases. Finally, we obtain Compatibility (enzyme) $=1 / 4=0.25<\operatorname{mincomp}$. Therefore, the biological databases are inconsistent in relation to the database attribute enzyme.

As for the latter case, $D B_{3}$ is ignored in the same way. The database attribute org of $D B_{1}$ and $D B_{4}$ is linked to Vertebrate concept in the ontology, and spec of $D B_{2}$ and $D B_{5}$ is linked to Organism concept in Figure 2. Among them, the spec attribute in $D B_{2}$ and $D B_{3}$ needs to be translated to the corresponding attribute specs in $D B_{5}$ for it is a systematic species name. Hence, the model $(A T T)=\{$ org, spec, org, spec $\}$. There are two possibilities by which to select the reference attribute here: (1) org; and (2) spec. If we use org as the reference attribute, we have Consistentset(org) $=\{$ org, org $\}$ with respect to $D B_{1}$ and $D B_{4}$, and Conflictset(org) $=\{$ spec, spec $\}$ in relation to $D B_{2}$ and $D B_{5}$. Therefore Compatibility(org) $=2 / 4=0.5 \geq$ mincomp. Therefore, the biological databases are consistent in respect to the queried database attribute animal : mouse using org. On the other hand, if we use spec as the reference attribute, we have Consistentset(spec) $=\{$ spec $\}$, Conflictset(spec) $=\left\{\right.$ org, org, spec $\left._{s}\right\}$ and Compatibility $($ spec $)=1 / 4=0.25<\operatorname{mincomp}$. Thus, they are inconsistent in respect to the database attribute (animal, mouse) using spec.

\section{Conclusions}

Knowledge acquisition from biological databases plays a nontrivial role in biological studies. However, the heterogeneity of biological databases has resulted in a significant lack of interoperability between them. The integration of biological databases is critical when dealing with heterogeneity but suffers from the twisted and deformed nature of biological data. Ontology-based integration of biological databases is an efficient way to capture knowledge from multiple sources. Nevertheless, no effort has been made to analyse the inconsistency in biological databases. This paper proposes a method to measure the inconsistency of biological databases via ontology. It assists in obtaining high quality data for data mining and knowledge discovery. We demonstrate our method by conducting experiments.

\section{References}

1. http://www.ncbi.nlm.nih.gov/.

2. Benson DA., Karsch-Mizrachi I., Lipman DJ., Ostell J. and Wheeler DL., GenBank Update, Nucleic Acids Research, vol 32 (Database issue), pp 23-26, 2004.

3. Stevens R., Goble C., Horrocks I. and Bechhofer S., OILing the Way to Machine Understandable Bioinformatics Resources, IEEE Trans Inf Technol Biomed, 6(2), pp 129-134, 2002. 
4. Williams N., Bioinformatics: how to get databases talking the same language, Science, 275(5298), pp 301-302, 1997.

5. Kohler J., Philippi S. and Lange M., SEMEDA: ontology based semantic integration of biological databases, Bioinformatics, 19(18), pp 2420-2427, 2003.

6. Philippi S. and Kohler J., Using XML Technology for the Ontology-based Semantic Integration of Life Science Databases, IEEE Trans Inf Technol Biomed, 8(2), pp 154-160, 2004.

7. Karp P.D., Riley M., Saier M., Paulsen I.T., Paley S.M. and Pellegrini-Toole A., The EcoCyc and MetaCyc Databases, Nucleic Acids Research, 30(1), pp 59-61, 2000.

8. Yeh I., Karp PD., Noy NF. and Altman RB., Knowledge acquisition, consistency checking and concurrency control for Gene Ontology (GO), Bioinformatics, 19(2), pp 241-248, 2003.

9. Kohler J., Philippi S. and Lange M., SEMEDA: ontology based semantic integration of biological databases, Bioinformatics, 19(18), pp 2420-2427, 2003.

10. http://www.geneontology.org/. 